\title{
The Collective Dynamics of Smoking in a Large Social Network
}

\section{Citation}

Christakis, Nicholas A., and James H. Fowler. 2008. The collective dynamics of smoking in a large social network. New England Journal of Medicine 358, no. 21: 2249-2258.

\section{Published Version}

http://dx.doi.org/10.1056/NEJMsa0706154

\section{Permanent link}

http://nrs.harvard.edu/urn-3:HUL.InstRepos:3710308

\section{Terms of Use}

This article was downloaded from Harvard University's DASH repository, and is made available under the terms and conditions applicable to Other Posted Material, as set forth at http:// nrs.harvard.edu/urn-3:HUL.InstRepos:dash.current.terms-of-use\#LAA

\section{Share Your Story}

The Harvard community has made this article openly available.

Please share how this access benefits you. Submit a story.

\section{Accessibility}




\title{
The Collective Dynamics of Smoking in a Large Social Network
}

Nicholas A. Christakis, M.D., Ph.D., M.P.H., and James H. Fowler, Ph.D.

\author{
A BSTRACT
}

\section{BACKGROUND}

The prevalence of smoking has decreased substantially in the United States over the past 30 years. We examined the extent of the person-to-person spread of smoking behavior and the extent to which groups of widely connected people quit together.

\section{METHODS}

We studied a densely interconnected social network of 12,067 people assessed repeatedly from 1971 to 2003 as part of the Framingham Heart Study. We used network analytic methods and longitudinal statistical models.

\section{RESULTS}

Discernible clusters of smokers and nonsmokers were present in the network, and the clusters extended to three degrees of separation. Despite the decrease in smoking in the overall population, the size of the clusters of smokers remained the same across time, suggesting that whole groups of people were quitting in concert. Smokers were also progressively found in the periphery of the social network. Smoking cessation by a spouse decreased a person's chances of smoking by $67 \%$ ( $95 \%$ confidence interval [CI], 59 to 73). Smoking cessation by a sibling decreased the chances by $25 \%$ (95\% CI, 14 to 35). Smoking cessation by a friend decreased the chances by $36 \%$ (95\% CI, 12 to 55 ). Among persons working in small firms, smoking cessation by a coworker decreased the chances by 34\% (95\% CI, 5 to 56). Friends with more education influenced one another more than those with less education. These effects were not seen among neighbors in the immediate geographic area.

\section{CONCLUSIONS}

Network phenomena appear to be relevant to smoking cessation. Smoking behavior spreads through close and distant social ties, groups of interconnected people stop smoking in concert, and smokers are increasingly marginalized socially. These findings have implications for clinical and public health interventions to reduce and prevent smoking.

From the Department of Health Care Policy, Harvard Medical School, Boston (N.A.C.); the Department of Medicine, Mt. Auburn Hospital, and the Department of Sociology, Harvard University - both in Cambridge, MA (N.A.C.); and the Department of Political Science, University of California, San Diego, San Diego (J.H.F.). Address reprint requests to Dr. Christakis at the Department of Health Care Policy, Harvard Medical School, 180 Longwood Ave., Boston, MA 02115, or at christakis@hcp.med.harvard. edu.

N Engl J Med 2008;358:2249-58. Copyright $(2008$ Massachusetts Medical Society. 
$\mathrm{R}$

OUGHLY 44.5 MILLION ADULTS WERE smokers in the United States in 2004, ${ }^{1}$ and smoking remains the leading preventable cause of death, ${ }^{2}$ with 440,000 deaths annually. ${ }^{3}$ Nevertheless, the prevalence of smoking has declined from $45 \%$ to $21 \%$ over the past four decades. $^{4}$

Past studies have documented the impact of dyadic social ties on the initiation and cessation of smoking, primarily in young people. ${ }^{5,6}$ However, the extent to which smoking depends on how people are embedded in a social network and the extent to which smoking behavior transcends direct dyadic ties are not known. Since diverse phenomena can spread within social networks, ${ }^{7-11}$ we conducted a study to determine whether smoking cessation also spreads more than from one person to another.

We evaluated a network of 12,067 people who underwent repeated assessments of their smoking behavior and social-network ties over a period of 32 years. We examined six issues: the existence of clusters of smokers and nonsmokers within the network, the association between one person's smoking behavior and smoking behavior in his or her social contacts, the dependence of this association on the nature of the social ties (i.e., ties between siblings, spouses, friends, coworkers, and neighbors), the influence of education and smoking intensity on the spread of smoking, the extent to which smoking cessation occurs in large subnetworks of people at one time, and the extent to which smokers move to the periphery of the social network across time.

\section{METHODS}

\section{SOURCE DATA}

The Framingham Heart Study was initiated in 1948, when 5209 people were enrolled in the original cohort. ${ }^{12}$ In 1971 , a total of 5124 children of the original cohort and their spouses were enrolled in the offspring cohort. ${ }^{13}$ In 1994 , a minority oversample of 508 people known as the "OMNI cohort" was initiated, and in 2002, the third-generation cohort, consisting of 4095 children of the offspring cohort and their spouses, was initiated. We analyzed data obtained from physical examinations and questionnaires performed during 3-year periods centered in 1973, 1981, 1985, 1989, 1992, 1997, and 1999 (see the Supplementary Appendix, available with the full text of this article at www.nejm.org). Validated measures of cigarette consumption were collected at each time point. ${ }^{14,15}$

\section{NETWORK ASCERTAINMENT}

For our study, we used the offspring cohort as the source of 5124 subjects (known as "egos" in the field of network science). Any persons to whom these subjects are linked - in any of the Framingham Heart Study cohorts - can, however, serve as social contacts (known as "alters"). A total of 12,067 subjects and contacts were connected at some point during the study period (1971 to 2003) (see the Supplementary Appendix), and connections were identified longitudinally. ${ }^{11}$ As a person's family changed because of birth, death, marriage, or divorce, and as their contacts changed because of residential moves, new employment, or new friendships, this information was recorded.

Overall, there were 53,228 observed family and social ties to the 5124 subjects, yielding an average of 10.4 ties per subject within the network (not including ties to neighbors). For example, 83\% of the spouses of subjects were in the network and $87 \%$ of subjects with siblings had at least one sibling in the network.

A total of $45 \%$ of the 5124 subjects were connected through friendship to another person in the network at some point. There were 3542 unique friendships, for an average of 0.7 friendship tie per subject. Because friendship identifications are directional, we studied three kinds of friendships: a "subject-perceived friendship," in which a subject identifies a contact; a "contact-perceived friendship," in which a contact identifies a subject; and a "mutual friendship," in which the identification is reciprocal. We hypothesized that the strongest interpersonal influence on subjects would occur in mutual friendships, followed by subject-perceived friendships, followed by contact-perceived friendships.

For $39 \%$ of the subjects, at least one coworker was captured in the network at some point. For $10 \%$ of the subjects, an immediate (nonrelative) neighbor was also present (more expansive definitions, such as living within $100 \mathrm{~m}$ of a subject, resulted in more neighbor ties but yielded similar results).

We included in the study only persons 21 years of age or older. At the inception of the study, 53\% of the subjects were women. The mean age of the subjects was 38 years (range, 21 to 70 ), and their 
mean educational level was 1.6 years of college (range, no education to $\geq 17$ years of education). Measures of occupational prestige of the subjects at each examination were available (see the Supplementary Appendix). ${ }^{16}$ Smoking among subjects in the Framingham Heart Study mirrored national trends; for example, among persons 40 to 49 years of age at each examination, the prevalence of smoking declined from $65.9 \%$ to $22.3 \%$ over the study period (see the Supplementary Appendix).

The study was approved by the institutional review board of Harvard Medical School and the board of the Framingham Heart Study. All subjects provided written informed consent.

\section{STATISTICAL ANALYSIS}

We treated smoking as a continuous variable in some analyses (e.g., number of cigarettes smoked per day), but we mainly used a dichotomous cut point of no cigarettes versus $\geq 1$ cigarette per day.

We graphed the network using the KamadaKawai ${ }^{17}$ algorithm and created a video of the network by means of the Social Network Image Animator (known as SoNIA) ${ }^{18}$ (see the Supplementary Appendix). The Kamada-Kawai algorithm positions nodes so that the nodes and the ties connecting them overlap as little as possible, thus producing interpretable images.

We measured the centrality of the subjects to assess whether they are near the center of the network. One measure of centrality is simply the number of a subject's ties; people who have more contacts tend to be more central. Eigenvector centrality, which was used in this study, takes this measurement a step further by weighting the contacts on the basis of how many other contacts each has. People who have "popular" contacts have higher centrality as compared with people who have less popular contacts (see the Supplementary Appendix). ${ }^{19}$

To study the clustering of smoking behavior, we compared the whole observed network at each examination to simulated networks with the same network topology and the same overall prevalence of smoking, but with the incidence of smoking randomly distributed across the nodes ("random smoking networks"). ${ }^{20}$ If clustering is occurring, then the probability that a contact is a smoker, given that a subject is a smoker, should be higher in the observed network than in the random networks. What we defined as the "reach" of the clusters is noted by identifying the point, in terms of a contact's degree of separation from any given subject, at which the probability that a contact is a smoker is no longer related to whether the subject is a smoker.

There are three explanations for clustering. First, subjects might choose to associate with contacts with similar smoking behavior (homophily). ${ }^{21}$ Second, subjects and their contacts might have common unobserved contemporaneous exposures that cause their smoking behavior to covary (confounding). Third, contacts might influence subjects (induction). Distinguishing induction from homophily is easier when longitudinal information about people's ties and attributes (i.e., smoking behavior) is simultaneously available. ${ }^{22}$

Hence, we specified longitudinal logisticregression models wherein the subject's smoking status (no cigarettes vs. $\geq 1$ cigarette per day) at a given time point $(t+1)$ was a function of attributes such as the subject's age, sex, education level, smoking status at the previous time point ( $\mathrm{t}$ ), and, most pertinent, the smoking status of his or her contacts at times $t$ and $t+1 .^{22}$ We used generalized estimating equations to account for multiple observations of the same subject across examinations and across subject-contact pairs. ${ }^{23}$ We assumed an independent working correlation structure for the clustered errors. ${ }^{23,24}$

The use of a time-lagged dependent variable (lagged to the previous examination) eliminated serial correlation in the errors (evaluated with a Lagrange multiplier test ${ }^{25}$ ) and also helped to control for a subject's genetic endowment and any intrinsic, stable predilection to smoke. The use of a lagged independent variable for a contact's smoking status helped to account for homophily. ${ }^{22}$ The key variable of interest was a contact's smoking behavior at time $t+1$. We found no statistically meaningful difference between the processes of smoking cessation and initiation in subjects depending on smoking cessation or initiation among their contacts; our models, therefore, evaluated concordance in change in smoking behavior (i.e., a contact starts smoking and the subject starts, or a contact stops smoking and the subject stops). Since cessation predominated in our time period, however, we phrase our results in terms of cessation.

We estimated these models in various subjectcontact pair types. We evaluated the possibility that unobserved exposures explained the associations by examining how the type or direction of 


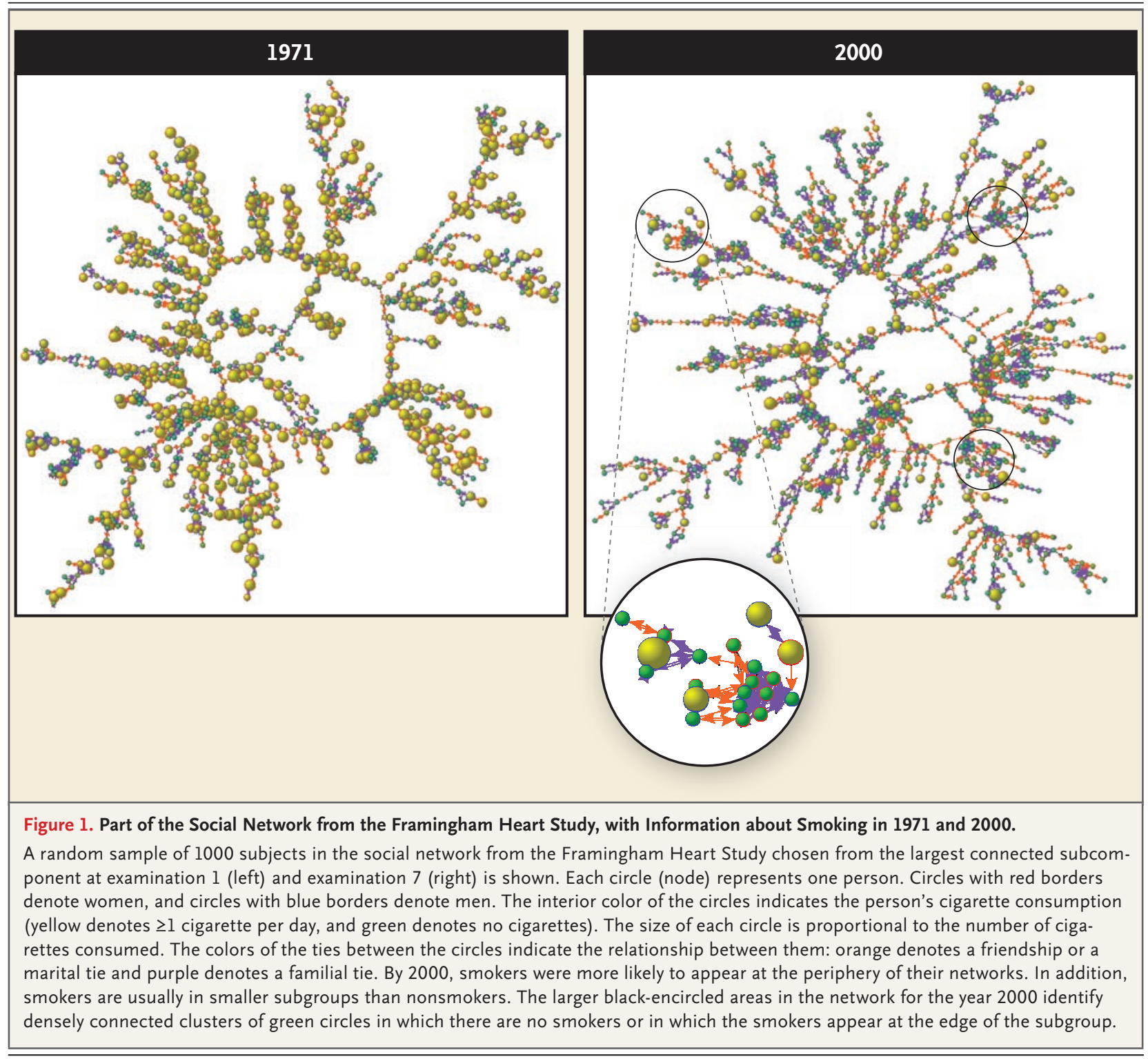

the relationship between the subject and contact affected the association between the smoking behavior of the subject and contact and by analyzing the role of geographic distance between subjects and their contacts.

We calculated $95 \%$ confidence intervals by simulating the first difference in the contact's contemporaneous smoking (changing from 0 to 1 ) and by using 1000 randomly drawn sets of estimates from the coefficient covariance matrix and assuming mean values for all other variables. ${ }^{26}$ All tests were two-tailed.

\section{RESULTS}

\section{NETWORK ANALYSIS}

Figure 1 depicts part of the network in 1971 and in 2000. There was a substantial change in the prevalence of smoking and in the social life of smokers during the period from 1971 to 2000 . In 1971, there were many more smokers as compared with 2000, and the smokers occupied the center of their circles of friends and family to the same extent that nonsmokers did. However, by 2000, most people had stopped smoking, and those who 


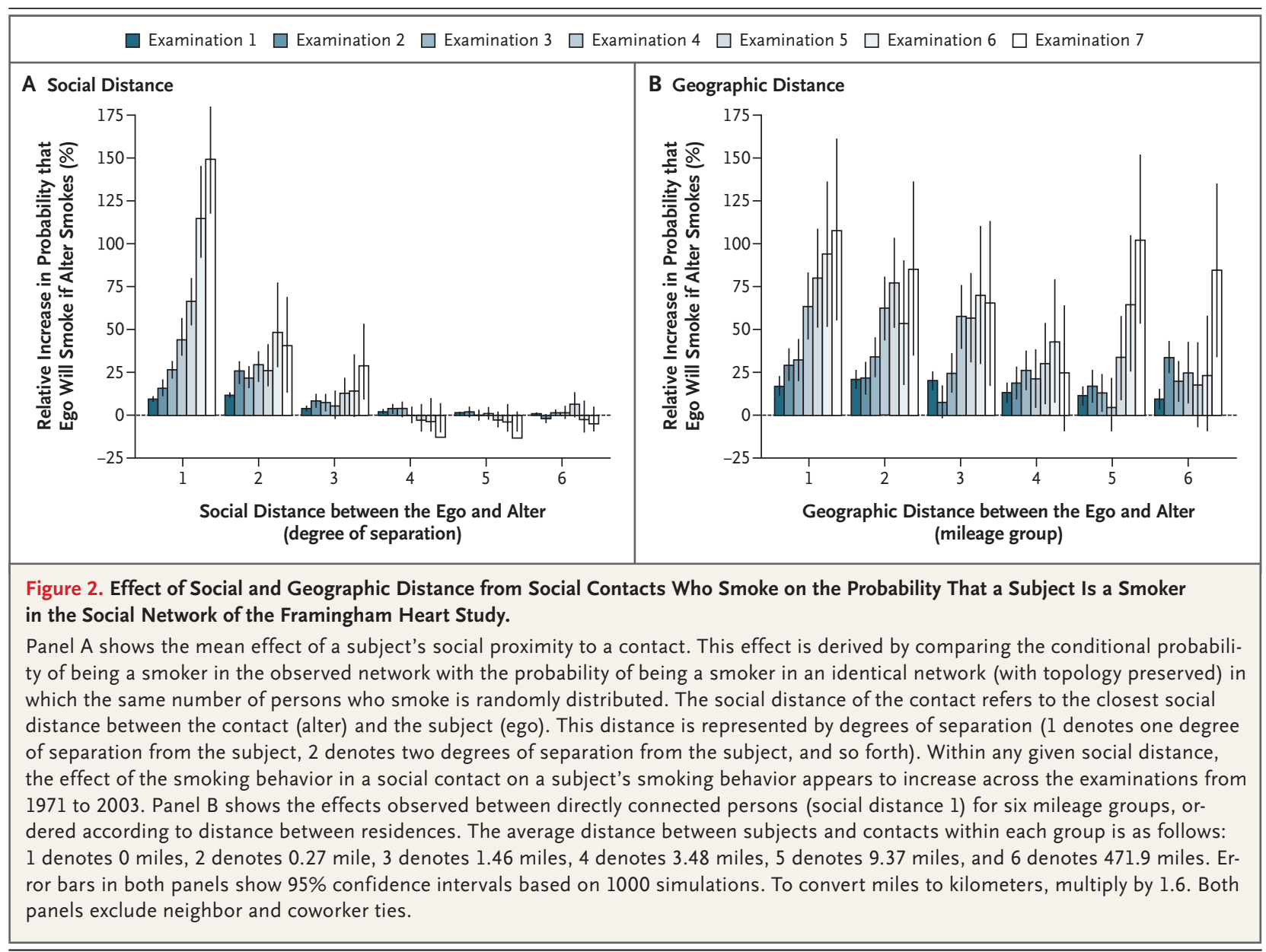

still smoked were more likely to be at the periphery of the network. Moreover, there was an increased tendency for smokers to be connected primarily to other smokers and for there to be relatively separate clusters of smokers and nonsmokers. Additional quantitative evidence is available in the Supplementary Appendix.

Figure 2A characterizes the clusters within the entire network more formally, and Figure $2 \mathrm{~B}$ characterizes the effect of geographic distance between subjects and contacts (see below). Across all of the examinations, the average risk of smoking among contacts who were connected to a subject who is a smoker (at one degree of separation) was $61 \%$ higher in the observed network than in a random network. The risk of being a smoker was also $29 \%$ higher for contacts' contacts (at two degrees of separation) and 11\% higher for contacts' contacts' contacts (at three degrees of separation).
By the fourth degree of separation, there is no excess relationship between a subject's smoking behavior and the contact's smoking behavior. Hence, on average, the reach of the smoking clusters was three degrees. Socioeconomic factors do not appear to explain this clustering (see the Supplementary Appendix).

Figure $3 \mathrm{~A}$ shows how the average size of a fully connected cluster of smokers has changed over time. It also shows the average size of these clusters that would have been observed if we kept the network topology and smoking prevalence the same and then randomly reassigned subjects to be smokers or nonsmokers. The observed cluster sizes were much larger than those expected because of chance, and they remained relatively stable as compared with the random networks, which declined sharply in size when prevalence decreased. These data provide evidence that suggests 
that people are not gradually stopping smoking at the fringes of clusters of smokers. Instead, it appears that whole clusters of connected smokers become nonsmokers together, causing the average cluster size to remain high even while overall prevalence declines (see also the video in the Supplementary Appendix).

Moreover, Figure 3B indicates that the observed centrality of smokers decreased over the 32-year period: smokers were increasingly more peripheral within the network, and nonsmokers were progressively more central. To assess whether this change might be caused by a higher mortality rate among smokers resulting in the severing of links between people who smoke, we restricted the analysis to persons who survived throughout the course of the study; the results were nearly identical (see the Supplementary Appendix). Additional analyses indicated that differences in education levels between smokers and nonsmokers did not cause these results (see the Supplementary Appendix). Moreover, we found that smoking tends to decrease a person's subsequent centrality, but centrality does not tend to decrease smoking (see the Supplementary Appendix).

\section{INTERPERSONAL MODELS}

We evaluated the extent of interpersonal association in smoking behavior with the use of regression analysis. Figure 4 summarizes these associations.

If a subject stated that a contact was his or her friend, the chance that the subject would be a smoker decreased by 36\% (95\% confidence interval [CI], 12 to 55) if the contact stopped smoking. Between mutual friends, the subject's risk of smoking decreased by $43 \%$ (95\% CI, 1 to 69). There was no significant effect of smoking cessation when the friendship was perceived by the contact but not by the subject (15\%; 95\% CI, -35 to 50 ).

Educational status appeared to be important among friends. When a subject had at least 1 year of college, the risk of the subject smoking decreased by $57 \%$ (95\% CI, 29 to 75 ) if the contact stopped smoking. When a contact had at least 1 year of college, the effect was similarly strong at 55\% (95\% CI, 26 to 74). Hence, persons with more education were both more influential and more able to be influenced. Among friends who both had at least 1 year of college, the chance that one person smoked decreased by $61 \%$ (95\% CI, 28
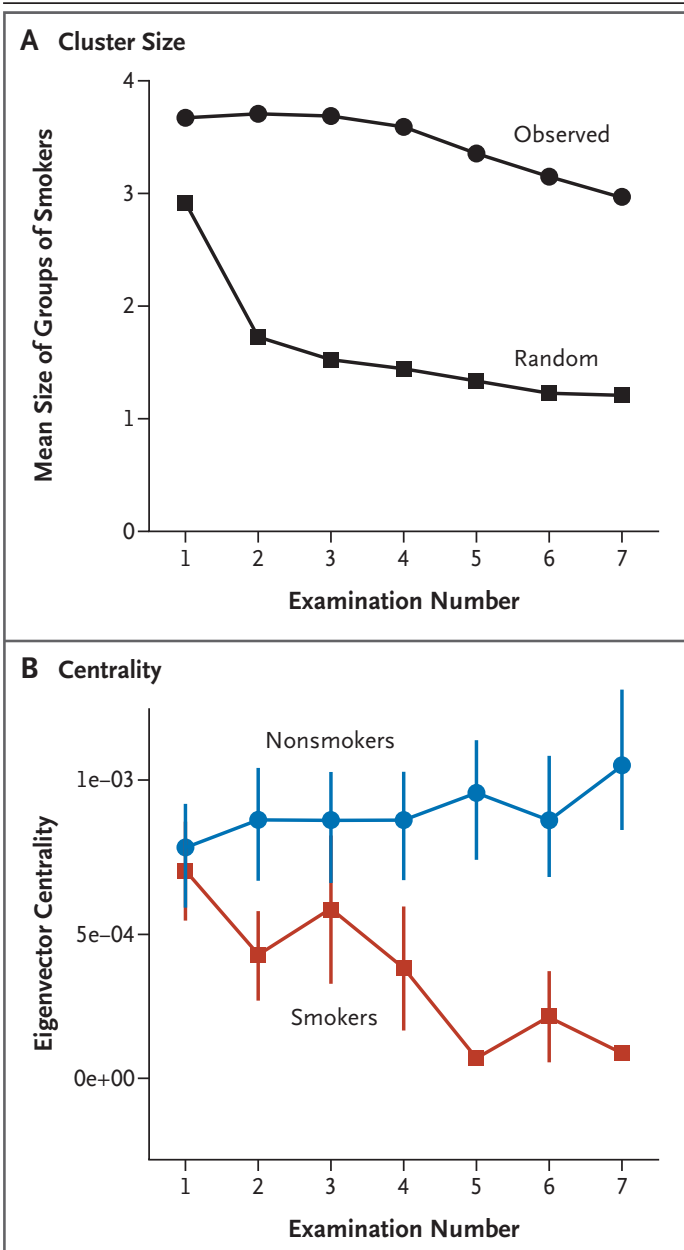

Figure 3. Cluster Size and the Centrality of Smokers across Time.

Panel A shows that smokers remained in tightly knit groups, even as the incidence of smoking sharply declined. Marginal smokers did not leave smoking groups; instead, whole clusters stopped smoking, and the clusters that did not maintained their previous size. The pattern in smoker cluster size that would have been observed if smokers were dropping out individually at random in the network is indicated by the lower line. Panel B shows Eigenvector centrality computed at each examination for smokers and nonsmokers. Although the centrality of nonsmokers remained roughly stable across all examinations, smokers became increasingly less central, and more peripheral, in the social network. Bars in both panels show $95 \%$ confidence intervals. In Panel A, confidence intervals are too small to see (the largest is slightly larger than the height of the dark squares). Both panels exclude neighbor and coworker ties.

to 81) when the other stopped smoking. Conversely, the association was not significant among pairs of friends in which at least one person had 
finished high school or had less than a highschool education. A model with an interaction term denoting pairs of friends with a high level of education showed that the association was significantly stronger $(\mathrm{P}=0.05)$ than the association in other kinds of friend pairs (see the Supplementary Appendix). Thus, the spread of smoking cessation among friends appears to be stronger through networks of persons who are highly educated. Educational status also appears to play an important role in peripheralization. Over the 32year period, the gap in centrality between smokers and nonsmokers widened more for persons with a high level of education than for persons with a low level of education (see the Supplementary Appendix). These data provide evidence that suggests that educated persons experience greater social marginalization due to smoking than less-educated persons.

Among pairs of coworkers, the effect of smoking cessation by one person was not generally a significant factor in smoking cessation by another person. However, most of our observations were based on coworkers at large firms who may not have known one another. When we restricted the analyses to smaller firms (those with up to six employees who participated in the Framingham Heart Study) we found that smoking cessation by a coworker increased the likelihood of smoking cessation by a subject by 34\% (95\% CI, 5 to 56). Moreover, the effect became stronger as the firm size decreased, as one might expect, since it is more likely that the subjects knew one another.

Among married couples, when one spouse stopped smoking, the other spouse was $67 \%$ ( $95 \%$ CI, 59 to 73) less likely to smoke; husbands and wives affected each other similarly. Among siblings, smoking cessation by one sibling increased the chance of smoking cessation by the other by $25 \%$ (95\% CI, 14 to 35). The smoking behavior of immediate neighbors did not have an effect on the smoking behavior of subjects.

We investigated the possible relevance of a number of other factors in additional models, including the size of groups of family members and friends, the prevalence of smoking in the subject's workplace, and the occupational prestige of the subjects. The association between the smoking behavior of contacts and subjects remained similar to the foregoing results (see the Supplementary Appendix).

We also examined the effect of geographic dis-

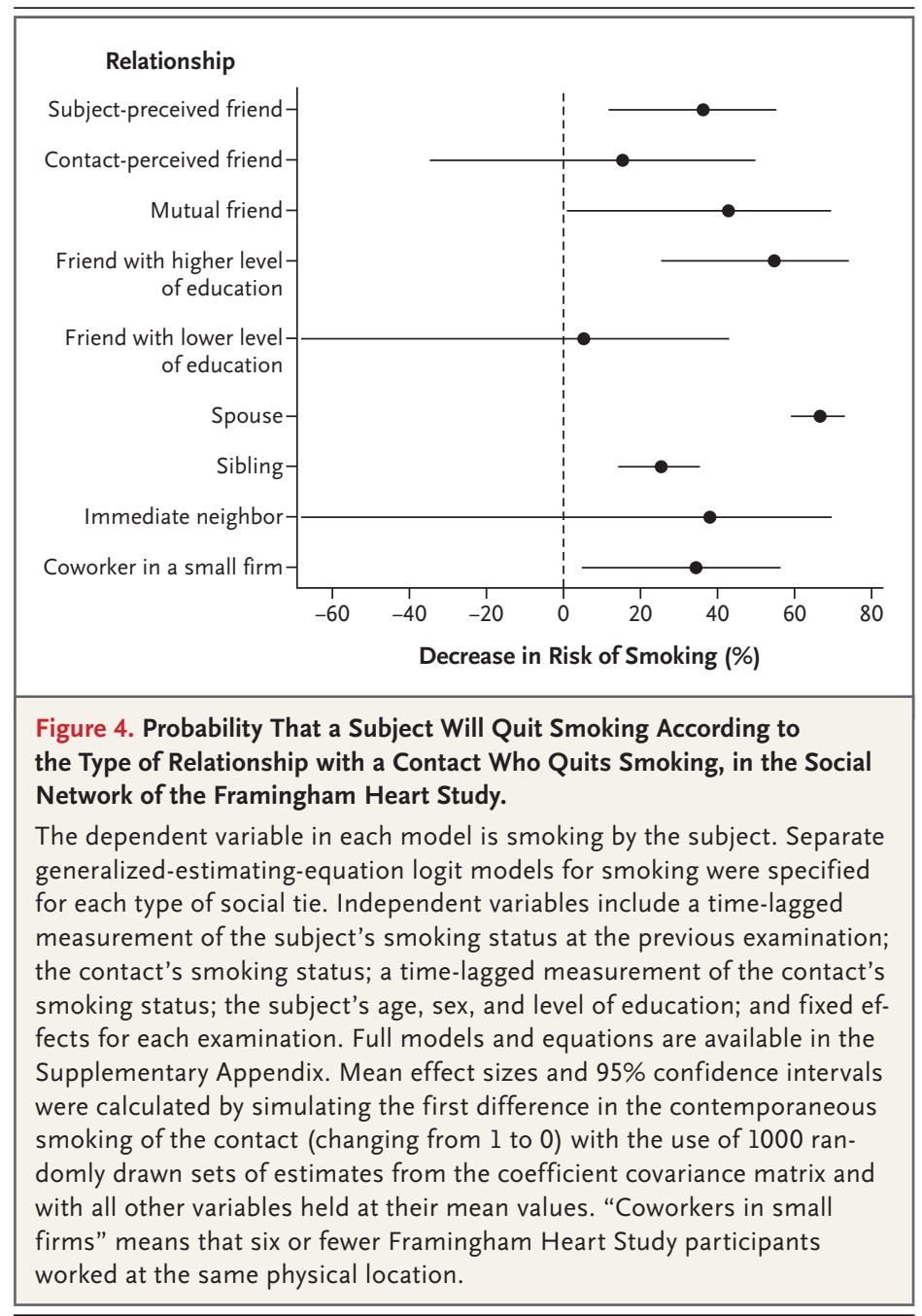

tance between subjects and contacts on the foregoing associations and found that distance did not modify the intensity of the effect of the contact's smoking behavior on the behavior of the subject. That is, smoking behavior was related between subjects and their contacts, regardless of how far apart they were geographically (Fig. 2B).

Finally, we examined the spread of different levels or intensities of smoking, changing the categorization of "smoker" in our models from persons who smoked at least 1 cigarette per day (casual smoking) to those who smoked at least 5 cigarettes (moderate smoking) or 20 cigarettes (heavy smoking). The association in smoking behavior between siblings and spouses remained strongly significant at all intensity levels. However, the association ceased to be significant between friends at heavy smoking intensities, and it also 
ceased to be significant between coworkers at both moderate and heavy intensities (see the Supplementary Appendix). Thus, it appeared that neither friends nor coworkers influenced heavy smoking as much as they did casual smoking.

\section{I SCUSSION}

The person-to-person spread of smoking cessation appears to have been a factor in the population-level decline in smoking seen in recent decades. Moreover, there appear to have been local smoking-cessation cascades, since whole connected clusters within the social network stopped smoking roughly in concert. This finding suggests that decisions to quit smoking are not made solely by isolated persons, but rather they reflect choices made by groups of people connected to each other both directly and indirectly at up to three degrees of separation. People appeared to act under collective pressures within niches in the network. As a further reflection of this phenomenon, persons who remained smokers were observed to move to the periphery of the network, ${ }^{27}$ and the network became progressively more polarized with respect to smokers and nonsmokers over the period from 1971 to 2003, with relatively fewer social ties between these groups.

We also found that the educational background of connected people mattered. The higher their education level, the more likely friends were to emulate each other with respect to smoking. In this regard, the diffusion of smoking cessation is consistent with the findings of previous studies of diverse innovations. ${ }^{28,29}$ This finding is also consistent with the idea that local social niches may arise within the network, and this emergence of niches may in turn contribute to the well-known socioeconomic gradient in smoking. That is, selfreinforcing norms may develop in groups of people, and these norms may augment individual decisions to quit. This process may lead to macrosocioeconomic patterns, similar to the adoption and spread of fashions first among high-status persons in society. ${ }^{28}$

Although connected persons might share an exposure to common environmental factors (e.g., cigarette taxes), the experience of simultaneous events (e.g., smoking-cessation campaigns in the workplace), or other common features (e.g., genes or sociodemographic attributes) that cause them to start or quit smoking simultaneously, our ob- servations suggest a process involving group dynamics and person-to-person spread. The grouplevel cessation of smoking was not solely due to people in the same household or workplace quitting together, since even contacts who were geographically separated evinced interpersonal effects and workplace effects seemed to depend on workplace size in a way that suggests that actual interpersonal contact is important. Moreover, the fact that immediate neighbors did not affect subjects helps to exclude joint exposure to local environmental factors (e.g., tobacco marketing, local taxes, or cigarette availability) as an explanation for our observations. Our models also controlled for a subject's previous smoking status; this helps to account for sources of confounding that are stable over time (e.g., childhood experiences, personality, or genetic endowment). Finally, our models controlled for the previous smoking status of contacts, thus helping to account for a possible tendency of smokers to form ties among themselves.

On the basis of both social theory and our previous studies on obesity, ${ }^{11}$ we expected that people would be more likely to emulate the behavior of friends they nominated rather than people who nominated them, and our results are consistent with this. Moreover, this pattern provides some evidence against the role of confounding, since any confounding factor would not respect such directionality in social ties.

Smoking behavior in contacts might influence smoking behavior in subjects by diverse biopsychosocial means, including changing the subject's norms about the acceptability of smoking, more directly influencing the subject's behaviors (e.g., a contact asking the subject not to smoke, or, conversely, a contact sharing cigarettes), or even fostering dependence through the inhalation of secondhand smoke. These mechanisms could not be distinguished on the basis of our data. We observed that geographic distance from a contact does not modify the effect of a contact's smoking behavior on a subject. This observation does suggest that social norms may be an important factor, since such norms may spread more easily over geographic distance than behavioral effects.

A change in the smoking behavior of more than one contact may be required for a subject to quit, and there may be additive or even threshold effects whereby a subject's probability of smoking cessation depends on smoking cessation by not one contact, but by two or more..$^{30}$ This phenom- 
enon may be especially likely in the case of smoking, since smoking is often deemed an explicitly social - and hence shared - behavior. Consequently, when a smoker runs out of easily available contacts with whom he or she can smoke, he or she may be more likely to quit. This possibility is also consistent with the group-level smoking cessation that we observed.

Network phenomena might be exploited to spread positive health behaviors. ${ }^{31-34}$ Indeed, cessation programs for smoking and for alcohol use that provide peer support - that is, that modify the person's social network - are more successful than those that do not. ${ }^{32,34}$ People are connected, and so their health is connected. ${ }^{35,36} \mathrm{Col}-$ lective interventions may thus be more effective than individual interventions. Moreover, medical and public health interventions to encourage people to quit smoking might be more cost-effective than initially supposed, since health improvements in one person might spread to others. ${ }^{35,37,38} \mathrm{Fi}$ nally, the isolation of smokers within social networks suggests that blanket policy approaches (e.g., advertising and taxation) may be usefully supplemented by interventions targeting small groups. In the case of smoking cessation in the past three decades, there is evidence of a cascade of salubrious behavior, and cessation of smoking in one person appears to be highly relevant to the smoking behavior of others nearby in the social network.

Supported by grants from the National Institutes of Health (NIH R-01 AG24448 and P-01 AG031093), by a Pioneer Grant from the Robert Wood Johnson Foundation, and by a contract from the National Heart, Lung, and Blood Institute (N01-HC-25195) to the Framingham Heart Study.

No potential conflict of interest relevant to this article was reported.

We thank Rebecca Joyce, Laurie Meneades, Molly Collins, Marian Bellwood, and Karen Mutalik for the expert assistance required to build the data set; and Joanne Murabito, M.D., and Emelia Benjamin, M.D., for advice.

\section{REFERENCES}

1. Cigarette smoking among adults United States, 2004. MMWR Morb Mortal Wkly Rep 2005;55:1121-4.

2. Department of Health and Human Services. Reducing the health consequences of smoking - 25 years of progress: a report of the Surgeon General. Atlanta: Centers for Disease Control and Prevention, 1989. (DHHS publication no. (CDC) 89-8411.)

3. Health United States 2003, with chart book on trends in the health of Americans. Hyattsville, MD: National Center for Health Statistics, 2003.

4. Faststats A to Z. Hyattsville, MD: National Center for Health Statistics. (Accessed April 22, 2008, at http://www.cdc. gov/nchs/fastats/Default.htm\#S.)

5. Chen PH, White HR, Pandina RJ. Predictors of smoking cessation from adolescence into young adulthood. Addict Behav 2001;26:517-29.

6. Powell LM, Tauras JA, Ross H. The importance of peer effects, cigarette prices and tobacco control policies for youth smoking behavior. J Health Econ 2005; 24:950-68.

7. Newman MEJ. The structure and function of complex networks. SIAM Rev 2003;45:167-256.

8. Bearman PS, Moody J, Stovel K. Chains of affection: the structure of adolescent romantic and sexual networks. Am J Sociol 2004;110:44-91.

9. Fowler JH. Turnout in a small world. In: Zuckerman A, ed. The social logic of politics: family, friends, neighbors, and workmates as contexts for political be- havior. Philadelphia: Temple University Press, 2005:269-87.

10. Crane D. Diffusion models and fashion: a reassessment. Ann Am Acad Polit Soc Sci 1999;566:13-24.

11. Christakis NA, Fowler JH. The spread of obesity in a large social network over 32 years. N Engl J Med 2007;357:370-9.

12. Dawber TR. The Framingham Study: the epidemiology of atherosclerotic disease. Cambridge, MA: Harvard University Press, 1980.

13. Feinleib M, Kannel WB, Garrison RJ, McNamara PM, Castelli WP. The Framingham Offspring Study: design and preliminary data. Prev Med 1975;4:518-25.

14. Patrick DL, Cheadle A, Thompson DC, Diehr P, Koepsell T, Kinne S. The validity of self-reported smoking: a review and meta-analysis. Am J Public Health 1994;84:1086-93.

15. Vartiainen E, Seppälä T, Lillsinde P, Puska P. Validation of self reported smoking by serum cotinine measurement in a community-based study. J Epidemiol Community Health 2002;56:167-70.

16. Treiman DJ. Occupational prestige in comparative perspective. New York: Academic Press, 1977.

17. Kamada T, Kawai S. An algorithm for drawing general undirected graphs. Inf Process Lett 1989;31:7-15.

18. Moody J, McFarland DA, Bender-deMoll S. Visualizing network dynamics. Am J Sociol 2005;110:1206-41.

19. Bonacich P. Factoring and weighing approaches to clique identification. J Math Sociol 1972;2:113-20.
20. Szabo G, Barabasi A-L. Network effects in service usage. (Accessed April 22, 2008, at http://lanl.arxiv.org/abs/physics/ 0611177.)

21. McPherson M, Smith-Lovin L, Cook JM. Birds of a feather: homophily in social networks. Annu Rev Sociol 2001;27:41544.

22. Carrington PJ, Scott J, Wasserman S. Models and methods in social network analysis. Cambridge, United Kingdom: Cambridge University Press, 2005.

23. Liang K-Y, Zeger S. Longitudinal data analysis using generalized linear models. Biometrika 1986;73:13-22.

24. Schildcrout JS, Heagerty PJ. Regression analysis of longitudinal binary data with time-dependent environmental covariates: bias and efficiency. Biostatistics 2005;6:633-52.

25. Beck N. Time-series-cross-section data: what have we learned in the past few years? Annu Rev Polit Sci 2001;4:271-93.

26. King G, Tomz M, Wittenberg J. Making the most of statistical analyses: improving interpretation and presentation. Am J Polit Sci 2000;44:341-55.

27. Strauss RS, Pollack HA. Social marginalization of overweight children. Arch Pediatr Adolesc Med 2003;157:746-52.

28. Pampel FC. Diffusion, cohort change, and social patterns of smoking. Soc Sci Res 2005;34:117-39.

29. Rogers EM. Diffusion of innovations. 4th ed. New York: Free Press, 1995.

30. Centola D, Eguiluz V, Macy M. Cascade dynamics of complex propagation. Physica A 2007;374:449-56. 
31. Wing RR, Jeffery RW. Benefits of recruiting participants with friends and increasing social support for weight loss and maintenance. J Consult Clin Psychol 1999;67:132-8.

32. Malchodi CS, Oncken C, Dornelas EA, Caramanica L, Gregonis E, Curry SL. The effects of peer counseling on smoking cessation and reduction. Obstet Gynecol 2003;101:504-10.

33. Brückner $\mathrm{H}$, Bearman P. After the promise: the STD consequences of adolescent virginity pledges. J Adolesc Health 2005;36:271-8.

34. McKnight AJ, McPherson K. Evaluation of peer intervention training for high school alcohol safety education. Accid Anal Prev 1986;18:339-47.

35. Christakis NA. Social networks and collateral health effects. BMJ 2004;329: 184-5.

36. Christakis NA, Allison PD. Mortality after the hospitalization of a spouse. N Engl J Med 2006;354:719-30.

37. Rosén M. On randomized controlled trials and lifestyle interventions. Int J Epidemiol 1989;18:993-4.

38. Cutler DM, Glaeser EL. Social interactions and smoking: NBER working paper no. W13477. Cambridge, MA: National Bureau of Economic Research, October 2007.

Copyright (C) 2008 Massachusetts Medical Society.

APPLY FOR JOBS ELECTRONICALLY AT THE NEJM CAREERCENTER Physicians registered at the NEJM CareerCenter can apply for jobs electronically using their own cover letters and CVs. You can keep track of your job-application history with a personal account that is created when you register with the CareerCenter and apply for jobs seen online at our Web site. Visit www.nejmjobs.org for more information. 